\title{
A New Unsupervised Feature Selection Method for Text Clustering Based on Genetic Algorithms
}

\author{
Pirooz Shamsinejadbabki, Mohammad Saraee*
}

\begin{abstract}
Nowadays a vast amount of textual information is collected and stored in various databases around the world, including the Internet as the largest database of all. This rapidly increasing growth of published text means that even the most avid reader cannot hope to keep up with all the reading in a field and consequently the nuggets of insight or new knowledge are at risk of languishing undiscovered in the literature. Text mining offers a solution to this problem by replacing or supplementing the human reader with automatic systems undeterred by the text explosion. It involves analyzing a large collection of documents to discover previously unknown information. Text clustering is one of the most important areas in text mining, which includes text preprocessing, dimension reduction by selecting some terms (features) and finally clustering using selected terms. Feature selection appears to be the most important step in the process. Conventional unsupervised feature selection methods define a measure of the discriminating power of terms to select proper terms from corpus. However up to now the valuation of terms in groups has not been investigated in reported works.

In this paper a new and robust unsupervised feature selection approach is proposed that evaluates terms in groups. In addition a new Modified Term Variance measuring method is proposed for evaluating groups of terms. Furthermore a genetic based algorithm is designed and implemented for finding the most valuable groups of terms based on the new measure. These terms then will be utilized to generate the final feature vector for the clustering process. In order to evaluate and justify our approach the proposed method and also a conventional term variance method are implemented and tested using corpus collection Reuters-21578. For a more accurate comparison, methods have been tested on three corpuses and for each corpus clustering task has been done ten times and results are averaged. Results of comparing these two methods are very promising and show that our method produces better average accuracy and F1-measure than the conventional term variance method.
\end{abstract}

Keywords Text clustering. Unsupervised feature selection. Genetic algorithm

\section{Introduction}

Up to the year 2000 it has been reported that well over one thousand petabaytes of data were accumulated and stored in mainframes, servers and client PCs not including Internet data. A significant portion of this vast amount of data is in text format (Sullivan 2001) and cannot be explored by humans unaided. Automatic tools for extracting useful knowledge from text are needed. Text has more complex structure than numeric data stored in database tables and therefore working with text requires more elaborate tools and techniques. Up to now, many applications of text mining are reported in the literature. Text mining is used in medical domains to find unknown relations between diseases and drugs. In the business world, text mining is utilized to extract useful knowledge from the web for better decision making. Furthermore to prevent threats against national security, Text Mining can be powerful tool for finding malicious patterns in texts that are exchanged between people.

Text mining covers many areas, including Information Retrieval (IR), Information Extraction (IE), Text Classification and Text Clustering. The last two are more significant due to their particular applications and 
normally are used as a subtask of other applications like IE and IR. Text classification means defining some common features between documents and then grouping them based on feature similarity (Miller 2005). The basic difference between text classification and text clustering is that in text classification, groups are predefined in spite of text clustering. Up to now many methods have been proposed in the field of text clustering including (Basu et al. 2002; Buddeewong and Worapoj 2005; Jain et al. 2004; Lee et al. 2006; Shang et al. 2006; Sun and Sun 2005; Wang and Zhang 2005; XU and Wang 2004). Some Text Classification methods also can be found in

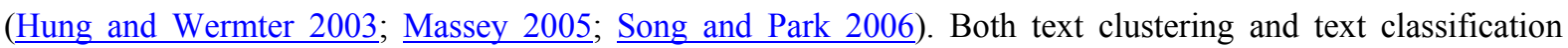
include three phases as shown in Figure 1.

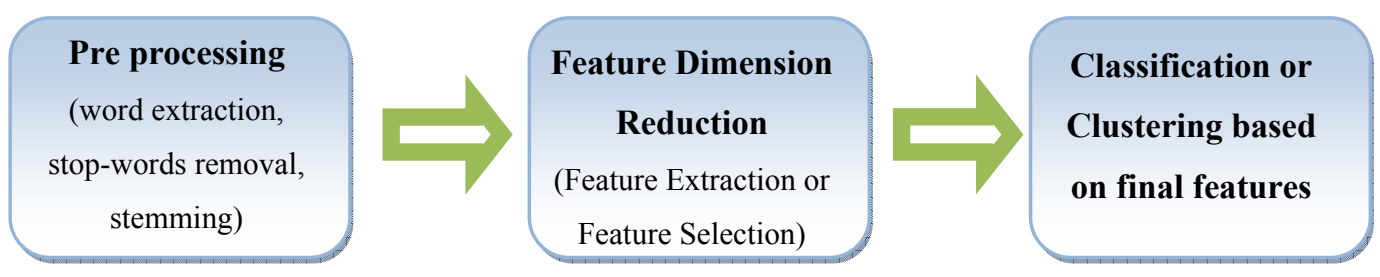

Figure 1: Phases in Text Classification and Text Clustering Process

The second phase of text clustering is feature dimension reduction. Since the dimension of a corpus usually is very large, text classification and clustering are prone to error and there is a need to reduce corpus dimension. Feature extraction and feature selection are two commonly used methods for reducing the dimension of corpus. It's important to notice that in text mining literature features usually are terms.

Feature extraction is the process of extracting new features from the set of all features by means of some functional mapping (Liu et al. 2005). Many works have been reported on feature extraction including those in (Bao et al. 2003; Kuntraruk and Pottenger 2001). The important shortcoming of feature extraction methods is that features which are created by these methods have no meaning and therefore it is hard to interpret the results (Liu et al. 2005). Feature selection methods on the other hand select some of the existing terms based on some measures and generate the final feature vector (Liu et al. 2005). Feature selection methods divide into two categories: supervised and unsupervised. Supervised methods need training data, unlike unsupervised methods. The work that is presented here is a new unsupervised feature selection method.

Up to now, many unsupervised feature selection methods have been reported in the literature. The most popular ones are Document Frequency (DF), Term Frequency Inverse Document Frequency (TFIDF), Term Contribution (TC), Term Variance (TV) (Liu et al. 2005; Yang and Pedersen 1997), Information Gain (IG), Mutual Information (MI), $\chi^{2}$, Relative Document Frequency (RDF), Relative Information Gain (RIG) and Relative Mutual Information (RMI) (Prabowo and Thelwall 2006; Yang et al. 2002; Yang and Pedersen 1997). All the methods mentioned work in three major steps:

1. Define a formula for measuring the discriminative power of a term.

2. Sort the terms based on the value of defined measurement.

3. Choose a number of the terms from top of the list.

As indicated above the discriminative power is the sign of the quality of a term for clustering or classification task.

In all traditional feature selection methods, the measure is defined for single terms only. The focal point of this work is to devise a new measurement for investigating discriminative power of a number of terms combined 
together as one and in relation with each other. The aim is to find and select terms with high discriminative power in the corpus even though they have low values in existing measurements. A GA method to compute the new measurement for terms is also presented.

The organization of the rest of this paper is as follows: section 2 introduces TV feature selection method. Our proposed feature selection method and Modified Term Variance measure (MTV) are presented in section 3. A genetic algorithm is designed for the new feature selection methods and is presented in section 4 . In section 5 experimental results of implementation of the new method and the traditional TV method on the reuters-21578 corpus are reported. Finally in section 6 we conclude the paper by outlining a few future extensions to this work.

\section{Term Variance Feature Selection Method}

This method computes the variance for all terms in documents and uses it as a measure for feature selection. The Term Variance method tries to find terms that have two criteria: 1- high document frequency and 2- nonuniform distribution among documents. Let $t_{i}$ be $\mathrm{i}^{\text {th }}$ term of corpus, $f_{i j}$ frequency of $t_{i}$ in $\mathrm{j}^{\text {th }}$ document and $\overline{f_{i}}$ average frequency of term $t_{i}$ in the corpus, then Term Variance is computed by this formula:

$$
v\left(t_{i}\right)=\sum_{j=1}^{N}\left[f_{i j}-\overline{f_{i}}\right]^{2}
$$

As shown above if term frequency is large but near to the average value among all documents, its variance decreases and the term has no chance of being selected.

\section{Proposed Feature Selection Method}

The proposed method is an unsupervised feature selection method and evaluates the discriminative power of terms in group form. The motivation behind this approach is that it is probable that some terms have low discriminative power for clustering but when they form a group, they may have good discriminative power. The reason is that when some correlated terms form a group together they represent a concept and this is what we are looking for in a clustering task. In addition we will solve the polysemy and synonymy problems with this method.

For example term "draft" has different meaning in the following categories:

1- document, proposal, white paper

2- conscription, military, war

3- withdrawal, cheque

Although "draft" may have quite high TV in the corpus, it has several different meanings and so can mislead the clustering process. On the other hand terms like "document" or "war" may have low TV and therefore are not chosen. The main point of this work is to discover terms like "war" or "document" in addition to "draft". Considering "draft" and "war" together in the feature vector can increase clustering accuracy because the term "war" distances documents that contain "draft" with the "proposal" meaning from documents that contain "draft" with the "war" meaning. 


\subsection{Vector Feature and Feature Vector}

In our new method we consider a vector of terms and evaluate its discriminative power combined together as a single feature; this group of terms is called vector feature. It's worth mentioning that vector feature is different from feature vector as used commonly in data mining literature. The feature vector is the final result of the dimension reduction phase in the Data Mining process but a vector feature is a type of feature that has more than one term. In our selection method first we find some vector features and combine them to form the final feature vector. Note that although they have the same format (i.e. group of terms) but their meanings are different.

\subsection{Modified Term Variance}

Since in the proposed method we intend to concentrate on groups of terms and evaluate their discriminative power, therefore the term variance measure is modified and adjusted to be employed by a vector feature. The Modified Term Variance (MTV) is computed using the following formula:

$$
\begin{gathered}
v\left(\vec{t}_{i}, \text { th }\right)=\sum_{j=1}^{N}\left[v f_{i j, t h}-\overline{v f_{i, t h}}\right]^{2} \\
\text { Where } \vec{t}_{i: \mathrm{i}^{\text {th }} \text { vector feature }} \\
\text { th : "contain threshold" } \\
\frac{v f_{i j, t h}: \text { frequency of } \vec{t}_{i} \text { in } \mathrm{j}^{\text {th }} \text { document where "contain threshold" is th }}{\overline{v f}_{i, t h} \text { : average frequency of } \vec{t}_{i} \text { in corpus where "contain threshold" is th }}
\end{gathered}
$$

The "contain threshold" shows the percentage of number of vector feature terms that must be in a document for vector feature to be considered as contained in that document.

$v f_{i j, t h}$ defines as follows:

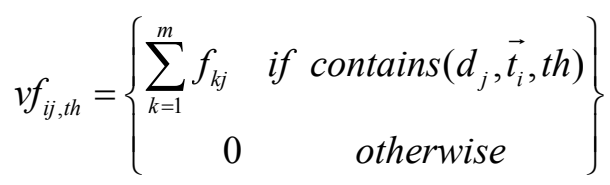

Where $f_{k j}$ : frequency of $k^{\text {th }}$ term of $\vec{t}_{i}$ in document $d_{j}$

$$
m \text { : number of terms in } \vec{t}_{i}
$$

$\operatorname{contains}\left(d_{j}, \vec{t}_{i}, t h\right)$ is a logical function that examines the presence of vector feature $\vec{t}_{i}$ in document $d_{j}$ with contain threshold $t h$. If the percentage of terms of $\vec{t}_{i}$ that are in document $d_{j}$ be equal or larger than $t h$ this function returns true and otherwise returns false.

Simply defined, the frequency of a vector feature in a document is the sum of the frequencies of its terms if the percentage of number of its terms presented in that document is at least equal to th and is zero otherwise. Average frequency for vector features is arithmetic mean of its terms.

Now we can use the MTV measure to find the most effective vector features for clustering. The problem here is that the number of possible vector features in a corpus is too many. The following example shows how this number may get too large. In a corpus with 100 documents where each document has 10 distinct terms, the number of possible vector feature will be 127400 . It is worth noting that in our computation we didn't count 
possible vector features that can be made by terms from different documents while we need to consider all of them for obtaining acceptable results. Therefore we couldn't use exhaustive searches for finding proper vector features. The GA which is described in the following section is a very well known approach to tackling this problem.

\section{Designed Genetic Algorithm}

GA is a learning method inspired by biological evolution. It tries to find the optimum or almost optimum points in search space without exploring all of it. Up to now some work has been done on using GA for text clustering. For example in (Song and Park 2006) GA is used in the clustering phase of the TC process shown in fig. 1 to finding the optimal number of clusters. Song and Park also have used a GA (Song and Park 2009) to devise a Latent Semantic Indexing method for an information retrieval task. In this work we exploit GA techniques for finding vector features with the most MTV value in the corpus.

\subsection{Search Space}

Each distinct term in the corpus is a dimension of search space. For example a corpus with 100 documents where each document has 10 distinct terms makes a search space with dimension of 1000 .

\subsection{Chromosomes}

In this work each vector feature is a chromosome. Because of the large dimension of the search space, use of binary encoding for chromosomes makes them too long and sparse (containing many $0 \mathrm{~s}$ and a few $1 \mathrm{~s}$ ). The chromosomes are illustrated as a set of terms, where each gene is a term. For example one chromosome could be like this: Oil . january. opec

\subsection{Initial population}

Each GA evolution starts with a population of initial chromosomes that form some initial solution to the problem. Here we first compute the number of chromosomes that will be generated from terms of each document using the following formula:

$$
\begin{gathered}
n o c_{j}=\left\lceil\left(l_{j} / L\right) \times p\right\rceil \\
l_{j}: \text { length of } \mathrm{j}^{\text {th }} \text { document } \\
L: \text { length of corpus } \\
p: \text { size of population }
\end{gathered}
$$

Then for each chromosome a random length will be generated and finally terms will be randomly selected from the document and form the chromosome.

\subsection{Fitness}

Here we defined fitness function as follows:

$$
\text { fitness }\left(\operatorname{ch}_{i}\right)=m t v\left(c h_{i}, t h\right) \times \ln \left(\text { length }\left(c h_{i}\right)+1\right)
$$




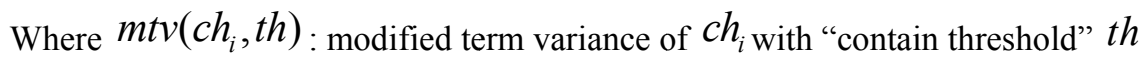

length $\left(c h_{i}\right)$ : number of genes(terms) in chromosome $c h_{i}$

In this formula, value ' 1 ' is added to the length of chromosome. This is to avoid the result of ln being zero in the case of chromosomes with length one, since we do not want to lose single terms with high term variance. It's worth noting that in our new method it is desirable to find not only single terms with high variance as in traditional methods but also groups of terms that can generate vector features with high MTV.

The subtle point to notice here is that the proposed fitness function might not cause the size of chromosomes in the evolution phase to grow dramatically, because when a chromosome becomes long, the probability that its terms co-occur in documents goes down and consequently its fitness will decline. This point is shown in figure 2 for an empirical experiment.

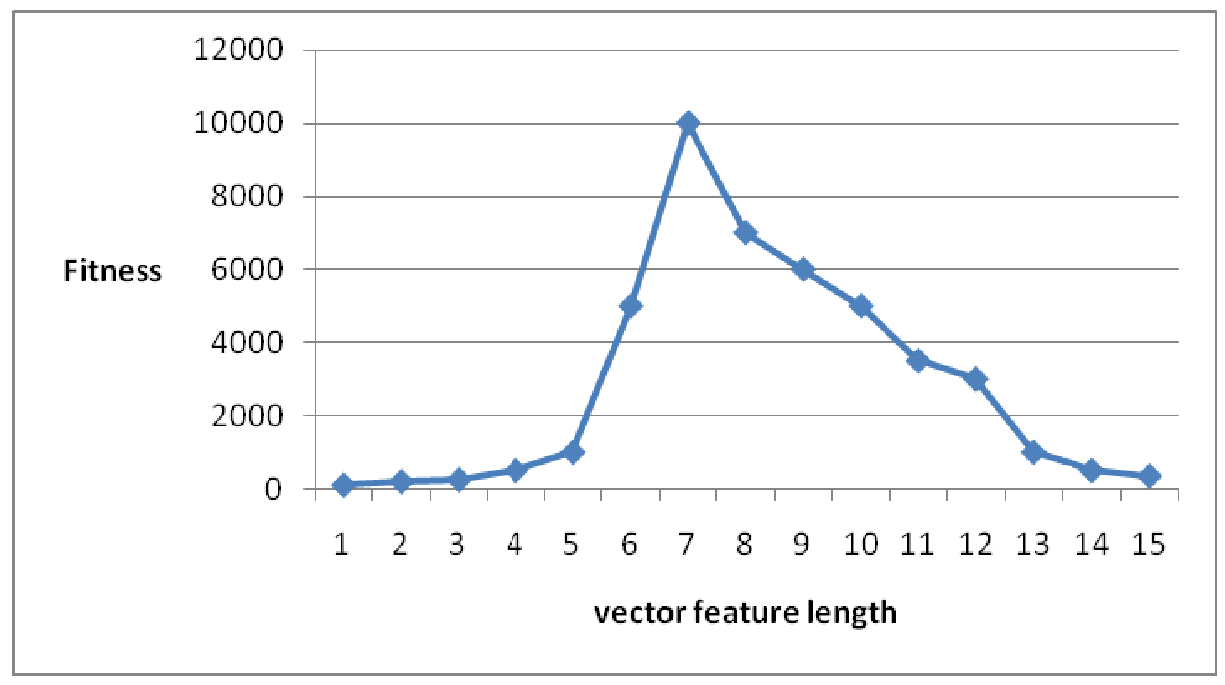

Figure 2: relation between vector feature length and its fitness

\subsection{Selection}

In the designed GA for selecting chromosomes from the population to add to the next generation the roulette wheel selection method (Goldberg 1989; Coley 1999; Mitchell 1997) is used. This method is commonly used in GA applications and the emphasis is on extraction in the search space more than exploration.

\subsection{Crossover operation}

Crossover is one of the most important operations in a GA that tries to reach a fitter generation than the current one by combining two chromosomes from the current generation. This operation makes GA different from a plain random search. The crossover operation used here is like a traditional single point crossover. It first selects two chromosomes by the roulette wheel selection method and then breaks them at random places uniformly and finally joins their parts in crossover fashion. This operation is shown in figure 3. 


\section{Create Crossover mask}

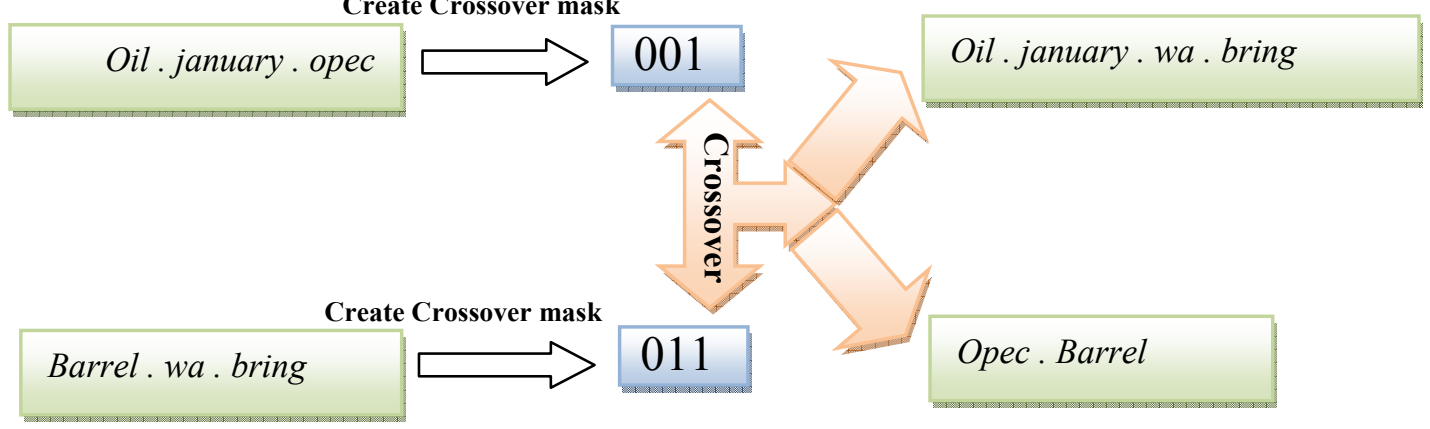

Figure 3: designed Crossover operation

\subsection{Mutation Operation}

Unlike the process of crossover, the role of mutation is to explore the search space. In other words this operation encourages the search process to go to unknown regions of search space. In this work we have used a traditional mutation operation (Coley 1999; Mitchell 1997). Mutation operation replaces a term of the feature vector that is randomly selected with another randomly selected term from the corpus as shown in figure 4 .

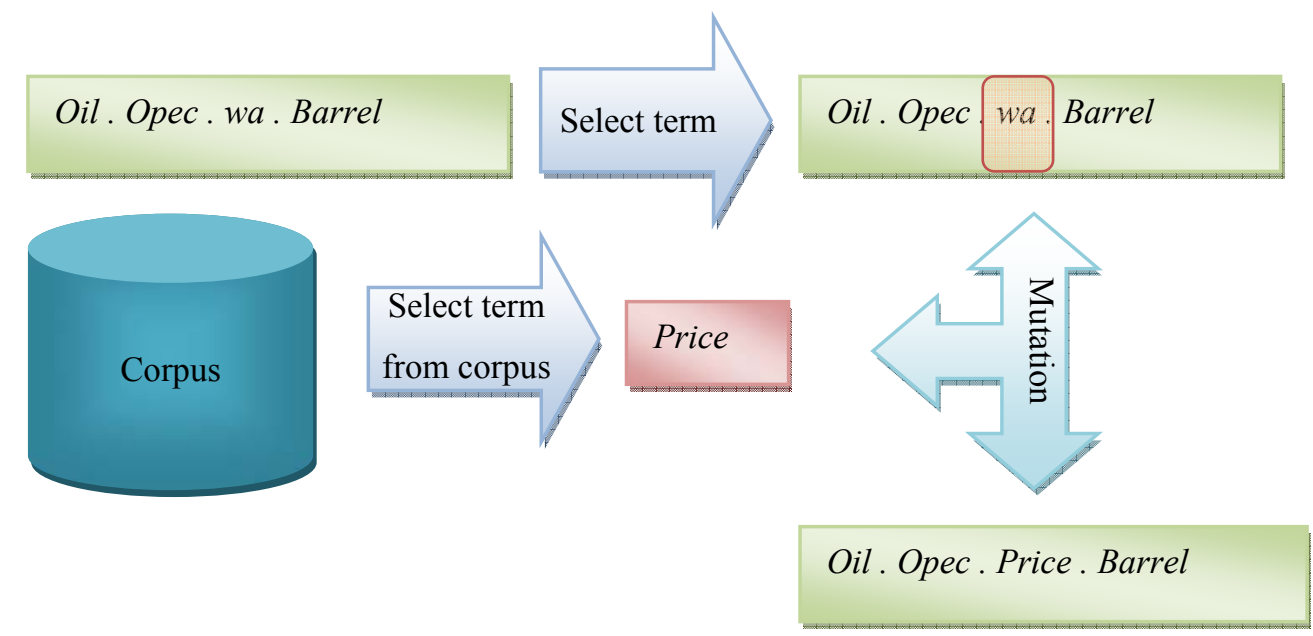

Figure 4: designed Mutation operation

\subsection{Niche Diversity}

In natural ecosystems, there are different niches for different species. These diverse niches are like optimum points in multimodal fitness functions (Beasley et al. 1993). In our feature selection method, the fitness function is multimodal and has some local optimum points. Each vector feature with relatively high MTV value is a local optimum point in the fitness function. There are some solutions for this problem that can be found in (Beasley et al. 1993). Here we have used the "sequential niche" solution. This method involves multiple runs of a GA and in each run a peak is found, selected and then remove from the search space (Beasley et al. 1993). Designed GA has been outlined in algorithm 1 . 
Algorithm 1: designed GA for new feature selection method

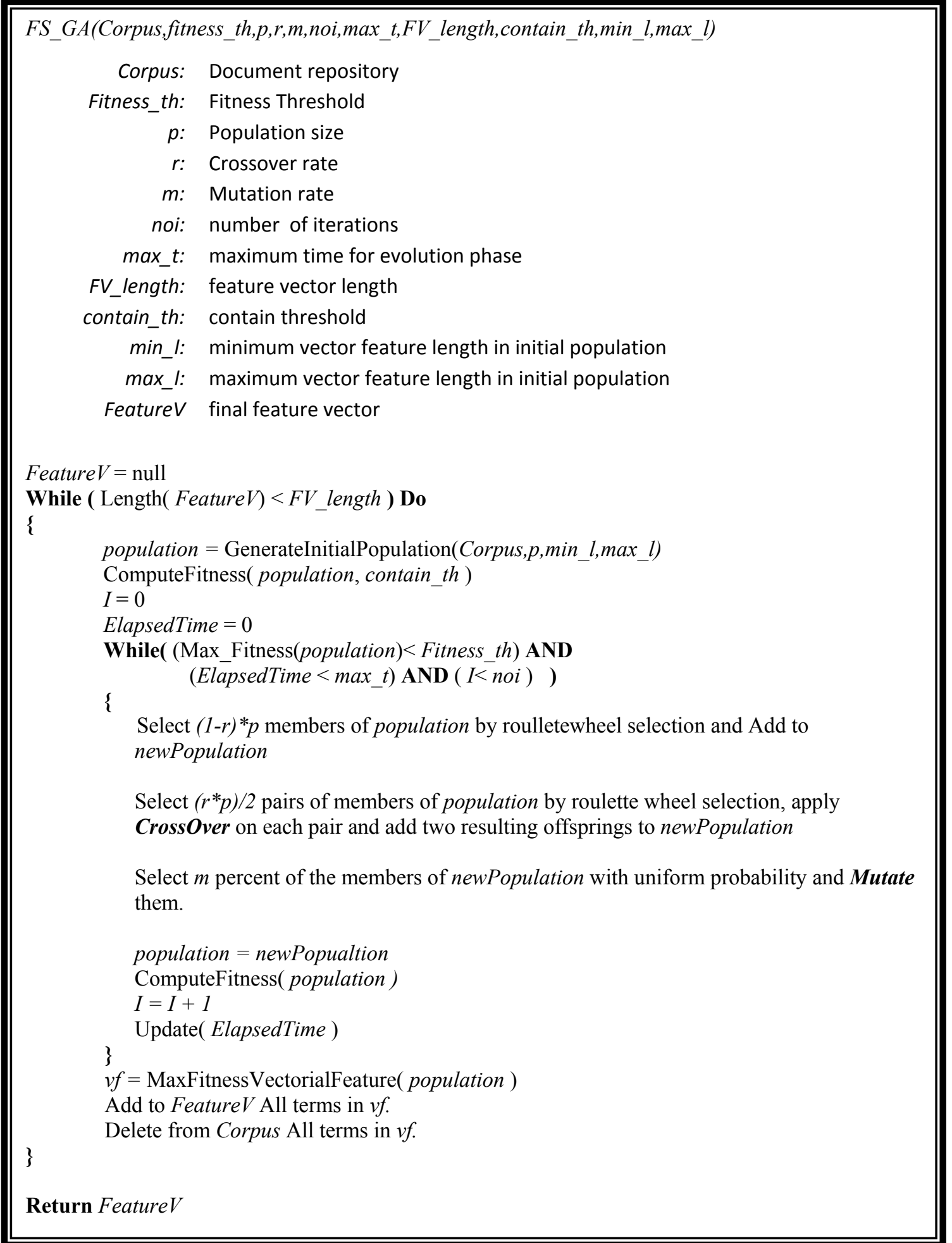

\section{Experimental Results}

Our proposed approach has been designed, implemented and tested on Reuters-21578 text collection distribution 1.0 (Reuters 2007). For the preprocessing phase of text clustering, we first extracted words from text and then removed stop words from it. For the stemming task the Porter algorithm has been used (Porter 2007). 
In this paper we have compared the traditional TV method and Document Frequency with our GA based method. It is shown in (Liu et al. 2005) that the TV method accuracy is as good as all other traditional unsupervised feature selection methods. We have used a combination of K-means and K-nearest neighbor methods for clustering. Clustering starts with the K-means method; then after clustering some predefined percent of documents $(20 \%$ in this case) the K-nearest neighbor method is used for the remaining documents. The value of $\mathrm{K}$ in the KNN method also increases adaptively based on the percentage of clustered documents. It is worth noticing that the presented clustering methods are used for all feature selection methods and therefore had no impact on comparison. In addition because the k-means method is sensitive to its start point therefore the start point for both text clustering methods is made equal in each experiment. The clustering phase is repeated 10 times for each feature selection method and an average of results is computed to provide a more precise comparison. To evaluate clustering performance we must count how many documents with the same topics are in the same cluster and also how many documents with different topics are in different clusters. For each pair of documents one of these states may hold (Liu et al. 2005):

ss: in our clusters and in the corpus both documents are placed in the same clusters.

sd: in our clusters both documents are placed in the same clusters but in corpus are in different clusters.

ds: in our clusters documents placed in different clusters but in the corpus are in the same clusters.

dd: in our clusters and in the corpus both documents placed in different clusters.

If the numbers of document pairs in the ss state are represented by $a$, sd by $b$, ds by $c$ and dd by $d$ then the average accuracy for Clustering is defined as follows:

$$
\text { AverageAccuracy }=\frac{1}{2} \times\left(\frac{a}{a+c}+\frac{d}{b+d}\right)
$$

Another measure for evaluating clustering is the F1-measure with the formula:

$$
\begin{gathered}
\text { F1Measure }=\frac{2 \times p \times r}{p+r} \\
\text { Where } p=\frac{a}{a+b} \\
r=\frac{a}{a+c}
\end{gathered}
$$

The maximum values of average accuracy and F1-measure are .5 and 1 respectively. These values could occur when all documents are clustered correctly.

Three methods including Term Variance, Document Frequency and our Modified Term Variance method were implemented and tested on three corpus of Reuters-21578 text collection. DF has been added because it is one of the basic methods. For a more accurate comparison each clustering task was performed 10 times and the results are averaged. The GA parameters have been learnt from experience as is shown in Table 1. 
Table 1: GA parameters

\begin{tabular}{|c|c|}
\hline Parameter & Value \\
\hline Contain_th & $70 \%$ \\
\hline min_l & 1 \\
\hline max_l & 4 \\
\hline Fitness_th & 100000 \\
\hline noi & 1000 \\
\hline max_t & $60 \mathrm{sec}$ \\
\hline$p$ & 1000 \\
\hline$r$ & 0.7 \\
\hline$m$ & 0.4 \\
\hline
\end{tabular}

Figure 5 shows the results for corpus reut2_001 relative to average accuracy. Also Figure 6 shows the results for corpus reut2_001 relative to F1-measure. Similarly Figure 7 and Figure 8 show the results for corpus reut2_002 and Figure 9 and Figure 10 shows the results for corpus reut2_003.

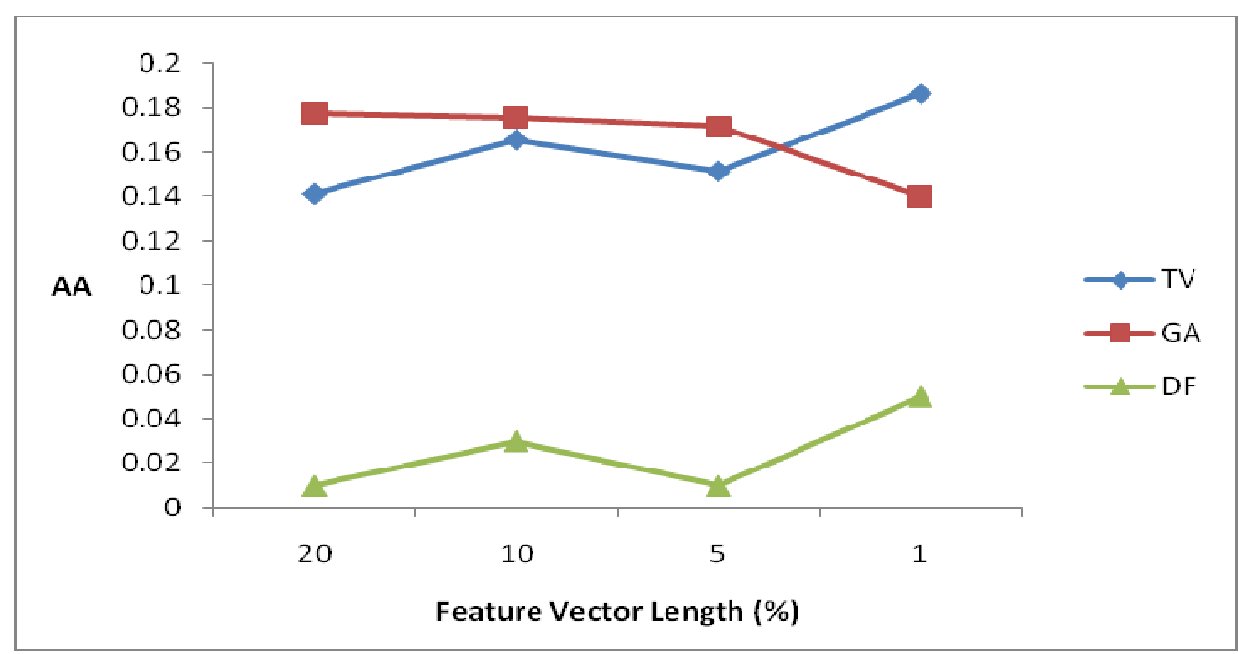

Figure5: Comparison of Average Accuracy on reut2_001

As shown in Figure 5 our proposed method has better average accuracy for three different feature vector lengths ( $20 \%, 10 \%$ and $5 \%$ of the length of corpus) on dataset reut2_001, although the TV method outperforms our method for feature vector with the length of $1 \%$. It is shown that DF method is the worse method as expected because it doesn't consider the variance of terms.

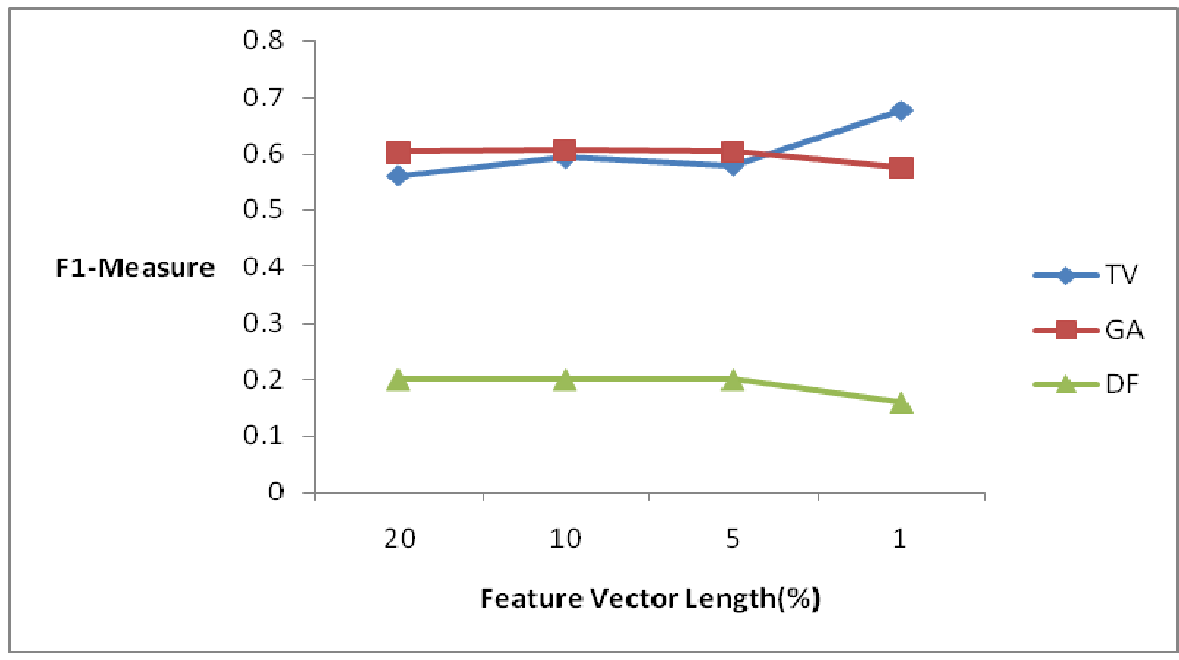

Figure6: Comparison of F1-Measure on reut2_001 
Figure 6 also confirms the result of Figure 5 in terms of F1-Measure. Unlike average accuracy, F1-Measure doesn't take into account the number of document pairs that are correctly placed in different clusters (true negative cases) and therefore there is a slight difference between the two measures. This difference shows that our method is more powerful in finding true negative cases than the TV method.

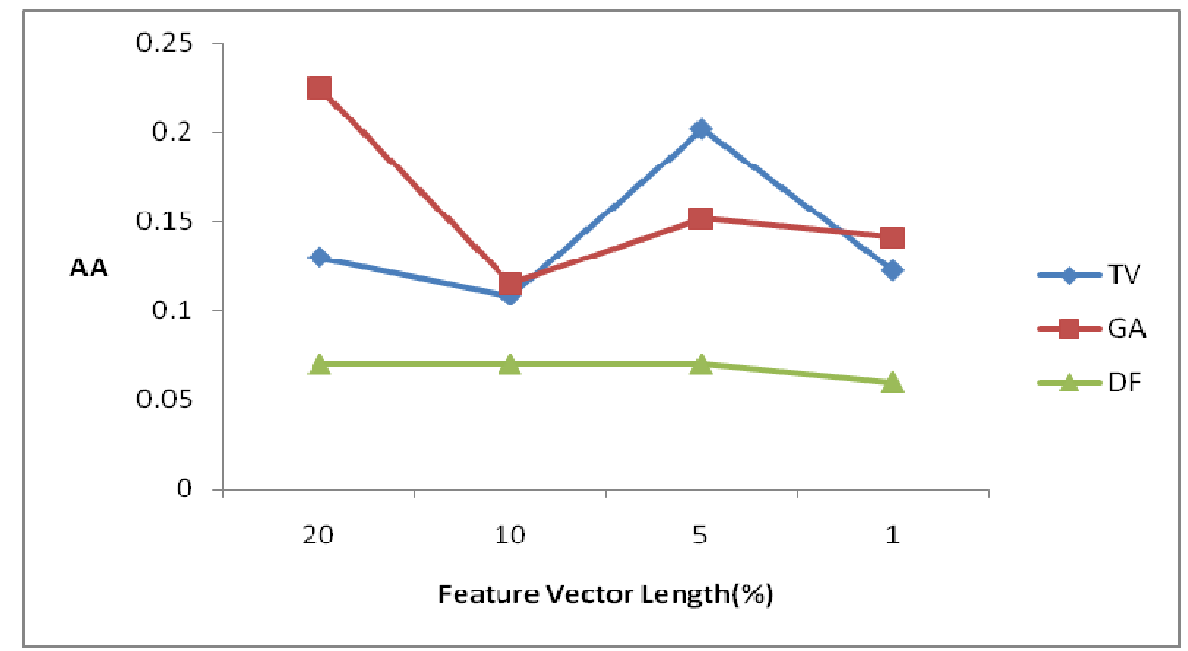

Figure7: Comparison of Average Accuracy on reut2_002

Figure7 shows the comparison of our proposed method with the TV and DF methods in terms of average accuracy on dataset reut2_002. As it is shown, average accuracy of GA and TV method fluctuates as the feature vector length changes. The reason may be as follows: some terms do not definitely belong to a specific concept. However, the frequencies of these terms in the corpus are close to the frequencies of some significance terms. Adding these terms to feature vector may disrupt the clustering task. These terms have all the possible frequencies. Consequently, with no training data calculating the optimum length of feature vector is impossible.

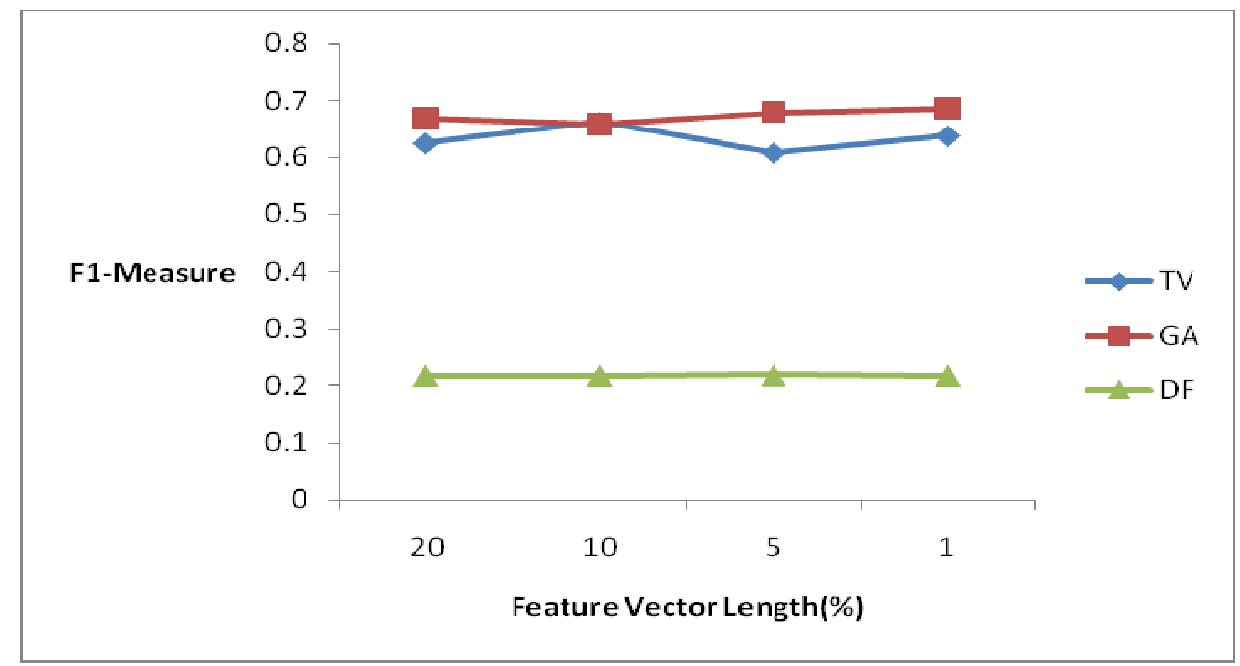

Figure8: Comparison of F1-Measure on reut2_002

Figure8 shows that the F1-Measure difference between the new method and the TV method is less than the average accuracy. This comes from the difference between F1-Measure and average accuracy in considering true negative cases. In addition, DF method results are very low but less sensitive to length of feature vector. 


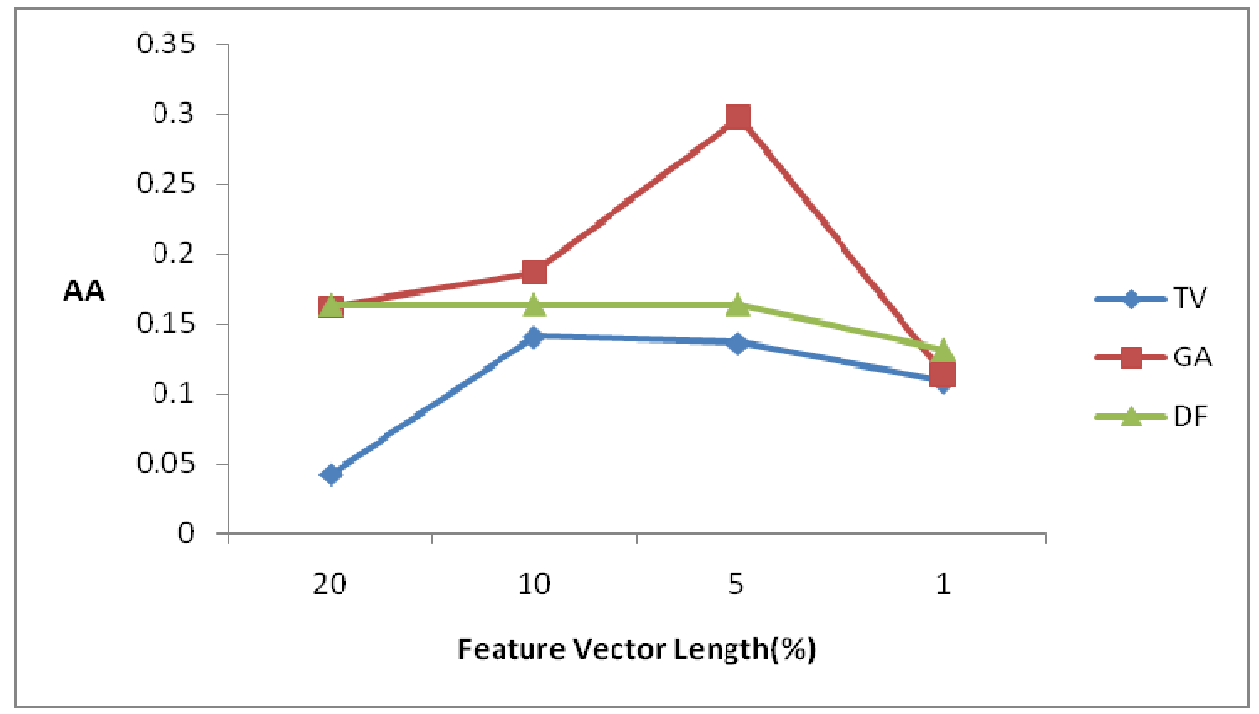

Figure9: Comparison of Average Accuracy on reut2_003

Figure 9 shows the result of comparison of the three methods on data set reut2_003. As it is shown the new proposed method attained the best average accuracy of all for almost all feature vector lengths. Also the DF method outperforms the conventional TV method in terms of average accuracy for all different lengths of feature vector.

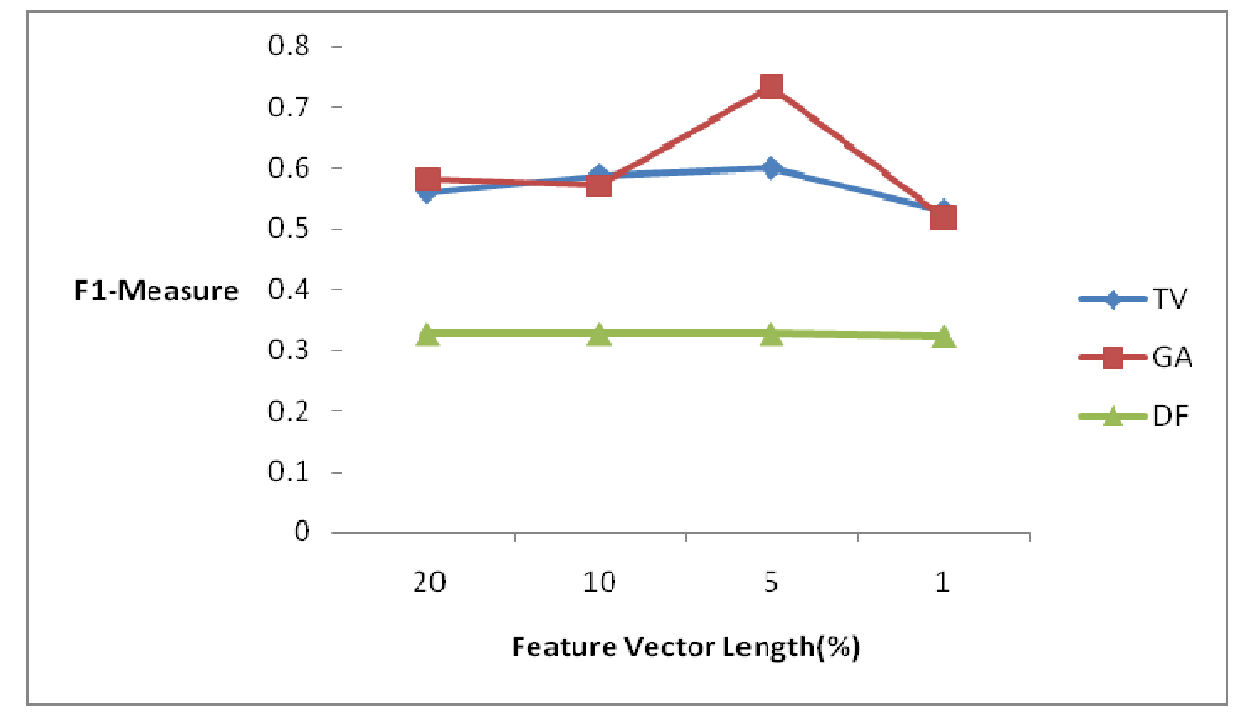

Figure10: Comparison of F1-Measure on reut2_003

In Figure10 F1-Measure of all methods are compared for dataset reut2_003. The results show that the new GA method and TV method have performances near to each other except for one feature vector length (5\%). It's worth noticing that the DF method gives a poor F1-Measure result especially in comparison to the TV method, though the DF method has better average accuracy. This indicates that the DF method could detect much more true negative cases on dataset reut2_003 than the TV method but these cases are ignored in F1-Measure computation.

In almost all comparisons the DF method got the worst results. This is predictable because DF method usually misleads on terms that are frequent and at the same time spread uniformly between documents. 
It is shown that the new method can reach better average accuracy and F1-Measure than TV and DF method in almost all points, although for a few feature vector lengths, the TV method outperformed our method. We have repeated the experiments 10 times and for three different corpuses; so that although the difference between our method and the TV method may not seem great, it proves that our method overall can reach better results than the TV method which is one of the most powerful methods in clustering as it is declared in (Liu et al. 2005).

The other interesting finding is that on average, half of the terms in feature vectors that were acquired by the new method and the TV method were different from each other. This shows that the new method can find and select new terms that are overlooked by the TV method. These terms have low term variance but when placed near another terms make vector features with high variances - and that it was our major motivation for devising this method.

As we expected any vector feature that came out from the GA contained terms belonged to the same concept. An example of a vector feature could be:

(Bank,USA, billion,loan,sale,hongkong,earn,store,expect)

In other words our method handles polysemy and synonymy automatically by grouping all terms belong to a concept.

The major weakness of the proposed method is that it is time-consuming. Using a GA for finding the most fit groups of terms is a time-consuming task. Finding feature vectors for clustering usually is an offline task, therefore the additional time imposed by the proposed method is not prohibitive. It is worth noticing that our method will automatically converge to the conventional TV method by finding one-term groups if TV can gain better results; but it will take much time for the GA and in our empirical results we have placed a time limit on GA.

\section{Conclusions and Future works}

In this paper a new method is presented based on using a GA for the unsupervised feature selection phase in a text clustering task. In our approach groups of terms are processed while in existing traditional methods each term is processed individually. We also presented a new Modified Term Variance measure for evaluating groups of terms in vector feature form. A GA based technique has been designed and implemented for finding vector features with high MTV and a sequential niche method combined with GA used for generating a final feature vector.

The result was that our method can find some terms neglected by the traditional TV method that can improve the clustering result. By comparing our proposed method with TV and DF methods on three corpus of Reuters21578 text collection it has been shown that our method outperforms existing methods on average accuracy and F1-Measure for almost all feature vector lengths. This proves that the feature selection method presented can find terms with higher discriminative power than the TV method.

The future work will be:

1. As is shown in the experiments, the accuracy of the methods are not similar for different feature vector lengths. This fact holds for all feature selection methods. We can find some feature vectors with different length in our method and then do text clustering based on those feature vectors separately and finally make a voting between results for each document. This solution can resolve the overfitting problem and at the same time maintain accuracy. 
2. We have used a concept called "contain Threshold" for defining the presence of a vector feature in a text. It indicates what percentage of terms of a vector feature must be in a document for it to be considered in that document. If this threshold is defined based on fuzzy sets theory, we may see better results.

\section{Acknowledgements}

We thank Pete Blindell for proofreading the manuscript.

\section{References}

Bao J, Shen J, Liu X, Song Q (2003) A new text feature extraction model and its application in document copy detection. In: Proceedings of the second international conference on machine learning and cybernetics. IEEE. pp 82-87

Basu A, Watters C, Shepherd M (2002) Support vector machines for text categorization. In: Proceedings of the $36^{\text {th }}$ Hawaii international conference on system sciences. IEEE

Beasley D, Bull D, Martin R (1993) A sequential niche technique for multimodal function optimization. Evolutionary Computation Journal. pp 101-105

Buddeewong S,Worapoj K (2005) A new association rule-based text classifier algorithm. In: Proceedings of the $17^{\text {th }}$ IEEE international conference on tools with artificial intelligence. pp 684-685

Coley D (1999) An introduction to genetic algorithms for scientists and engineers. World scientific.

Goldberg D (1989) Genetic Algorithms in Search, Optimization and Machine Learning. Kluwer Academic Publishers.

Hung C, Wermter S (2003) A dynamic adaptive self-organising hybrid model for text clustering. In: Proceedings of the third IEEE international conference on data mining (ICDM'03). pp 75-83

Jain G, Ginwala A, Aslandogan Y (2004) An approach to text classification using dimensionality reduction and combination of classifiers. In: Proceedings of the IEEE international conference on information reuse and integration (IRI). pp 564-569

Kuntraruk J, Pottenger M (2001) Massively parallel distributed feature extraction in textual data mining using HDDI. In: Proceedings of the $10^{\text {th }}$ IEEE international symposium on high performance distributed computing. pp 363-370

Lee C, Yang H, Ma S (2006) A novel multilingual text categorization system using latent semantic indexing. In: Proceedings of the first international conference on innovative computing, information and control. IEEE

Liu L, Kang J, YU J, Wang Z (2005) A comparative study on unsupervised feature selection methods for text Clustering. In: Proceeding of NLP-KE. Vol. 9, pp 597-601

Massey L (2005) Real-world text clustering with adaptive resonance theory neural networks. In: Proceedings of IEEE international joint conference on neural networks (IJCNN'05) 5:2748-2753

Miller T (2005), Data and text mining a business applications approach. Prentice Hall, New York.

Mitchell T (1997) Machine Learning. McGraw-Hill, Washington

Prabowo R, Thelwall M (2006) A comparison of feature selection methods for an evolving RSS feed corpus. In: Information processing and management $\mathrm{J}$ 42:1491-1512 
Porter algorithm, http://tartarus.org/ martin/PorterStemmer, cited on 2007

Reuters-21578 text collection, http://kdd.ics.uci.edu/databases/reuters21578/reuters21578.html, cited on 2007

Shang W, Qu Y, Haiban Z, Houkuan H, Yongmin L, Hongbin D (2006) An adaptive fuzzy knn text classifier based on gini index weight. In: Proceedings of the $11^{\text {th }}$ IEEE international symposium computers and computers and communications (ISCC'06)

Song W, Park S (2006) Genetic algorithm-based text clustering technique: automatic evolution of clusters with high efficiency. In: Proceedings of the seventh international conference on Web-Age information management workshops (WAIMW). pp 17-25

Song, W, Park S (2009) Genetic algorithm for text clustering based on latent semantic indexing. Computers \& Mathematics with Applications, 57(11-12): pp 1901-1907.

Sullivan, D., Document warehousing and text Mining, John Wiley, New York, 2001.

Sun F,Sun M (2005) A new transductive support vector machine approach to text categorization. In: Proceedings of NLP-KE. IEEE, pp 631-635

Wang B, Zhang S (2005) A novel text classification algorithm based on naïve bayes and KL-divergence. In: Proceedings of the sixth international conference on parallel and distributed computing, applications and technologies (PDCAT’05). IEEE

XU J, Wang Z (2004) A new method of text categorization based on PA and kohonen network. In: Proceedings of third international conference on machine learning and cybernetics, Shanghai, pp 1324-1328

Yang S, Wu X, Deng Z, Zhang M, Yang D (2002) Relative term-frequency based feature selection for text categorization. In: Proceedings of the first international conference on machine learning and cybernetics, Beijing. IEEE. pp 1432-1436

Yang Y, Pedersen J (1997) A comparative study on feature selection in text categorization. In: Proceedings of ICML-97, 14th international conference on machine learning. pp 412-420 